\title{
Nickel Complexes of Alanine Having a Preference for One Chiral Form over Another upon Crystallization
}

\author{
Awni Khatib and Fathi Aqra \\ Department of Chemistry, Faculty of Science and Technologw. Hebron Chiversity. P. O. Box 40 . Hebron. \\ West Bank, Palestine. * E-mail: an'm5lahotmail.com \\ Recened Mav 25, 2009, Accepted Jhl 26, 2009
}

\begin{abstract}
Three complexes, [bis(L-, D- and DL-alaninato)(diaqua)]nickel(II) di-hydrate, were prepared and characterized by X-ray crystallography, gas chromatography, UV-Visible spectroscopy and isothermal calorimetry. Small deviations from the 50:50 distribution of the enantiomers assigned to chiral preferences of the ligands in the complexes were observed. The surprising and unexpected results indicate that complexing alanine with nickel(II) ion alters the racenization rates of $\mathrm{D}$ and $\mathrm{L}$ isomers of the amino acid. The precipitated complex due to change in $\mathrm{pH}$ resulted in preferential precipitation of one isomer with respect to the other. It has been observed that this altemation is ognented by the long time span which would result in preferential protein foming from the L-isomer. Although the results are bizarre and perplexing, ther are fascinating and sound scientifically.
\end{abstract}

Key Wonds: Racemization. Alanine complexes. Chirality

\section{Introduction}

Amino acids are very necessary in biological bodies, and complex readily with the first transition series ions such as nickel(II) ion. There is a wealth of information on the structures of transition metal complexes with amino acids (including nickel and alanine) in the literature. The subject of homochirality in living systems is so controversial. challenging and an open question. It was proposed that the original selection was at random, then continues. Different racemization rates were observed for D-and L-alanine solutions compared to D- and L-alanine when present in solutions as nickel conjugates. ${ }^{1}$ The differences were sufficient to lead to enantiomeric enhancement when DL alanine was allowed to crystallize after racemization treatment. These results may provide important clues to the origin of biochirality. There are no physical or chemical differences between enantiomers except for their interaction with polarized light. Therefore. reported differences between enantiomers are typically attributed to experimental error or trace impurities. Unexpected differences between D and $\mathrm{L}$ enantiomers of amino acids. peptides and sugars have recently been investigated. ${ }^{2-3}$ The differential rates of $D$ - and L-ty rosine crystallization was also reported. ${ }^{8}$

Nickel and iron are the most abundant transition metals in the earth's cnist. At the time of life's origin. their ionic forms were present as solutes in the early earth's oceans. It seems likely that amino acids in solution would, therefore. be present as metal complexes rather than as free amino acids. Furthermore. it is expected that any amino acid synthesized by abiotic reactions would be racemic. Even if there were some process by which enantiomeric enhancement occurred. racemization would tend to return the enhanced species to racemic mixtures. However. if the enhancement occurred during racemization. this might provide a continuous source of anino acids with small enantiomeric enhancement. Such a phenomenon has been observed in several amino acids present in the Murchison meteorite. with enhancements ranging up to $8 \% \mathrm{~L}$ enantiomers. ${ }^{g-12}$ It has also been observed that small enhancements can influence chemical reactions that produce chiral products. For instance. ${ }^{1 s}$ it has been reported that there is a strong tendency for $D$ sugars to be produced when they were synthesized from DL-glyceraldehy de in the presence of L-amino acids. Previous studies of chiral enhancement in racenuic nixtures were based on pure amino acids. and the effect of metal complexes on such processes is virtually unexplored. Therefore, this paper describes results of experiments that demonstrate differences in racemization rates and the determination of possible differences in electronic structures of the nickel(II) complexes that could lead to measurable stuctural alterations. A chirality preference for alanine coordination to nickel(II) is reported, and the paper focuses on the interesting concept that small, but significant changes in bonding lead to selective precipitation of one isomer, and presents compelling evidence of these changes. The differences in bond distances used to rationalize energy differences are within the standard deviation of the measured value since they are small and may or may not be statistically significant. It is. therefore. not possible to comment exactly on the factors affecting the selective precipitation. As such. the best that can be said is that one isomer selectively' precipitates for unknown reasons, and a proposition may be made

\section{Experimental}

Racemization of nickel-alanine complexes. Solutions of DL-alanine ( $10 \mathrm{~mL} .0 .01 \mathrm{M}$ ) and nickel chloride hexalydrate or nickel carbonate $(5 \mathrm{~mL}, 0.01 \mathrm{M})$ were mixed in a $2: 1$ ratio. The $\mathrm{pH}$ was adjusted to 9.0 by dropwise addition of $\mathrm{KOH}$ solution $(0.0 \mathrm{l} \mathrm{M})$. and the complex formation was monitored by UV-Vis spectrophotometer. The resulting solution $(5.0 \mathrm{~mL})$ was transferred into Pyrex glass tubes $(0.80 \times 10.0 \mathrm{~cm})$ sealed at one end. after which the tubes were sealed at the other end. 
The tubes were, then. placed in a constant temperature sand bath set at $150^{\circ} \mathrm{C}$ for 240 hours. A blue solution and a blue precipitate were obtained. The crystals (reaction vessel 2) were separated from the solution (reaction vessel 1). A sample of aqueous solution of DL-alanine was exposed to the same conditions (reaction vessel 3 ). ${ }^{14}$ Similar experiment was carried out with nickel carbonate and treated as described above. A stoichiometric amount of sodium sulfide solution $(5 \mathrm{~mL})$ was added to each of the three reaction vessels, which results in removal of the nickel(II) ion as an insoluble nickel sulfide. and release of alanine from the complex. After 24 hours. the black nickel sulfide was precipitated. and all three solutions were transferred to new $10 \mathrm{~mL}$ reaction vessels. They were dried under nitrogen and derivatized to N-TFA-amino acidisopropyl ester for analy sis by gas chromatography.

Gas chromatograph and chiral separation. Gas chromatograph was used to measure the extent of racemization of alanine by separating the $N$-TFA-amino acid-isopropyl ester derivatives on a $150 \mathrm{ft}$. X 0.02 in stanless steel capillary column coated with 50.50 mixtures of $N$-docosanoyl-L-valyl-t-butamide and $\mathrm{N}$-octadecanoyl-L-ralyl-cyclohexyl ester. A flame ionization detector was employed. Baseline separation of the D- and L-alanine peaks was obtained. ${ }^{14}$

Spectral shift measurements. The UV-Visible spectra were measured with a Hewlett-Packard UV-Vis spectrophotometer. The green hexa-aqua nickel(II) complex, at $\mathrm{pH}=6.0$. shown absorption maxima at 302,382 and $644 \mathrm{~nm}$. while the blue bis-alaninato-diaqua-rickel(II) complex. at $\mathrm{pH}=8.5$. shown absorption maxima at $302,370.5$ and $618.5^{1}$.

Crystallization conditions. Crystallization of D- or L-alaninato nickel(II) complexes was induced either by concentrating the solution under nitrogen or by standing for days. Two kinds of crystals were produced: Pale blue prism-like and blue rhombic. respectively. Small amounts of blue crystals were also produced during the racemization reaction at the bottom of the tubes. with a clear blue solution remaining on top. The concentration of the nickel-alanine complex was supersaturated after the racemization treatment. so that few crystals appeared in the solution spontaneously probably due to the change in $\mathrm{pH}$ upon heating. These crystals were analyzed for their $\mathrm{D}$ and L-alanine ratios and provided a convenient way to contrast the $D: L$ ratios in solution and in the solid.

$\mathrm{X}$-ray diffraction. The crystal stnictures (allocated deposition numbers CCDC 718340,7183+1 and 716012) and the ply sical data of the three nickel(II) complexes with D. L and DLalanine were reported previously by us and others in a view to observe possible differences related to chiral influences. ${ }^{16.31}$

Isothemal calorimetry. Enthalpy changes were measured at $30^{\circ} \mathrm{C}$ with an ITC (VP-ITC Micro Calorimeter by Micro Cal, LLC. Northampton. MA-USA). The reactants were nickel(II) ion and D-. L- or DL-alanine solutions. at $\mathrm{pH} 8.0$. and the products were [bis(D-, L-and DL-alaninato)diaqua]nickel(II) dihydrate. Concentrations of the alanine (L. D and DL) were varied from $100 \mathrm{mM}$. $50 \mathrm{mM}$. $5 \mathrm{mM}$ and $0.25 \mathrm{mM}$, and that of the nickel(II) ion was constant at $100 \mathrm{mM}$. The nickel(II) ion (3 microliter aliquots) was injected into $2.0 \mathrm{~mL}$ of the alanine solution in the instrument cell. Injections were repeated 10 times at 3 minute intervals and the heat of reaction was recorded when the same value was measured in 5 or more injections.

\section{Results and Discussion}

This paper describes. in general, the chiral preference in the process of metal complexation with amino acids. The $\mathrm{D}$ and $\mathrm{L}$ alanine produced two well-separated peaks in the gas chromatograms. The retention times. area $\%$ and ratio $\%$ values are given in Tables $1-3$. When the solution of the DL-alaninato complex was subjected to the racemization conditions. a precipitate was detected. When the whole sample (solution + precipitate) was analyzed. the $D: L$ ratio was almost $1: 1$. On the other hand. when the solution is separated from the precipitate. the analysis reveals that the precipitate has significantly more D-form while the opposite is true for the solution. Therefore, it may be claimed that either the $\mathrm{D}$-alaninato nickel complex undergoes racenuzation at a higher rate than $L$-alaninato nickel complex, so that there is a trend toward increasing L-alanine in solution and increasing D-alanine in the precipitate, or the D-complex precipitates out preferentially. When the L-alanine complex was racemized. there was a clear difference between

Table 1. As control four racemization experiments were performed at different time intervals at $\mathrm{pH}=8.2$. Temperature $=122$ for the pure D.L-alanine solution

\begin{tabular}{cccc}
\hline Time(hrs) & No. of Samples & \%D-isomer & Ratio \\
\hline 0 & 2 & 50.0 & $1: 1$ \\
72 & 2 & 50.3 & $1: 1$ \\
120 & 2 & 50.4 & $1: 1$ \\
240 & 2 & 50.3 & $1: 1$ \\
\hline
\end{tabular}

Table 2. Racemization of nickel-alanine complexes

\begin{tabular}{cccccc}
\hline Compund & $\begin{array}{c}\text { Physical } \\
\text { Fom }\end{array}$ & $\begin{array}{c}\text { No. } \\
\text { Samples }\end{array}$ & Time & $\begin{array}{c}\text { Average } \\
\text { D/L }\end{array}$ & DRatio \\
\hline D,L- & sol'n + ppt & 3 & 72 & 49.4 & $0.988: 1$ \\
& sol'n $+p p t$ & 3 & 240 & 49.3 & $0.986: 1$ \\
D.L- & ppt & 4 & 72 & 52.9 & $1.06: 1$ \\
& ppt & 4 & 120 & 65.3 & $1.31: 1$ \\
& sol'n & 4 & 120 & 44.2 & \\
L & sol'n + ppt & 4 & 240 & 19.8 & \\
& ppt & 4 & 240 & 38.2 & \\
\hline
\end{tabular}

Table 3. The concentrations of $\mathrm{D}$ and $\mathrm{L}$ upon allowing the complex DL-Bis-alaninato diaquanickel(II) to form a precipitate without heating. It is shown that the D complex is less in solution while it is more in the precipitate

\begin{tabular}{lccc}
\hline Solution & RT & Area\% & Ratio\% \\
\hline D-alanine & 27.85 & 44.3 & 99.4 \\
L-alanine & 28.70 & 44.6 & 100.0 \\
\hline Precipitate & RT & Area\% & Ratio\% \\
\hline D-alanine & 27.75 & 35.4 & 100 \\
L-alanine & 28.61 & 34.7 & 98 \\
\hline
\end{tabular}


the solution and the precipitate which resulted during the racemization process. As a result. the precipitate contains more D isomer than the solution. This difference is significant because the lattice energy for the complexed D-isomer is more exothermic during the reaction of nickel with alanine. The bonds of the $\mathrm{D}$-isomer are, therefore. expected to be shorter and stronger. This is contrary to the expectation that there should be no energy difference between optical isomers. The UV-Visible spectroscopy and the X-ray structural analysis for the complexes would support this observation. The bond length diferences are summerized in Table 4 . The heat of reaction of the complex formation was measured for all three complexes using ITC which gave no definitive differences between the three complexes

It is shown (Table 2) that when the solution and precipitate were derivitized and analyzed, it gave almost a 1:1 ratio. but when the precipitate was separated and both were derivatized separately. the D-isomer concentration is much higher: $\mathbf{5 2 . 9 \%}$ and $65.3 \%$. We have observed considerable enrichment of the nickel-alanine complexes by D-isomer in the precipitate and by the L-isomer in the supernatant (Table 2). However. it is not clear if such behaviour holds in a statistically meaningful series of experiments. In principle. spontaneous enrichment of the precipitate by one of the isomers in a single experiment is not too unusual. Therefore, stable reproduction of the same sign of enrichment would be a ground breaking sensation. All other indications of differences between the enantiomeric complexes. such as differences in crystallographic parameters are well within the experimental errors.

The nickel-alanine complexes in solution readily formed excellent crystals. It would not be expected that there would be any observable differences in the crystal structures of the different nickel-alanine complexes isomers. Examination of the crystal structures revealed that the average $\mathrm{Ni}-\mathrm{N}$ bond in the $\mathrm{L}$-isomer is longer than its counterpart in the $\mathrm{D}$-isomer by $0.24 \mathrm{pm}$ (or $0.48 \mathrm{pm}$ per complex molecule) which gives -0.80 $\mathrm{kj} /$ mole in favor of the crystallization of the D-isomer which was not observed in the measured heat of reaction. ${ }^{16.18}$ There is a small apparent difference of the bond distances between the D \& L isomers. However. statistically they are identical. though we beleive that a small trend over a long peroid of time would make this difference significant.

UV-Vis spectrophotometry was used to determine whether there are shifts in imax that would be consistent with the observed differences in racemization rates. It is well known that such shifts are related to bond strength. If the nickel(II) atom with $\mathrm{D}$-alanine has a stronger coordinate bond than that with L-alanine this should be reflected by the shifts when the

Table 4. Bond Length differences (pmin) between D and L complexes with nickel ions

\begin{tabular}{lll}
\hline L $-D$ & for Ni-O3 & $=+0.11 \mathrm{pm}$ \\
L $-D$ & for Ni-O1 $=$ & $-0.12 \mathrm{pm}$ \\
L-D & for Ni-N1 $=+0.34 \mathrm{pm}$ \\
L-D & for Ni-N2 $=+0.14 \mathrm{pm}$ \\
\hline Total & & $+0.47 \mathrm{pm}$ \\
\hline
\end{tabular}

two enantiomers are compared. At $\mathrm{pH}=6.0$, the absorption maxima were at 367 and $602 \mathrm{~nm}$ for the $\mathrm{L}$-alanine complexed isomer. These maxima undergone some shifts in the nickel complex with D-alanine isomer (Table 5). However. the peaks for D-alanine were blue-shifted I - 3 nu compared to L-alanine. which is consistent with a greater bond strength in the D complex.

The heats of reactions measured by ITC differs for the three reactions at the same conditions. The heat of reaction of $L-$. and DL-alanine in the formation of [bis(D-. L- and DL-alaninato)diaqua]nickel(II) complexes are -14.3 to $-15.1 \mathrm{kj} / \mathrm{mole}$. This indicates that there are statistically significant differences. since the heats of reactions of the L- and D-conplexes are not similar.

We have shown from X-ray studies that the crystal structure of each complex isomer is unique. Complexes formed from different enantiomers of alanine show some minor differences in bond lengths and angles. The findings show that the D-complex precipitates preferentially more than the L-complex. This unusual anomaly may be due to different energies of the bonds formed between the alanine isomer and nickel(II) aton. The percentage of the D-isomer in the precipitate formed after racemization was significantly enhanced over the percentage of the D-isomer in solution. This was observed when we started with the L-isomer of the nickel-alanine complex, and was further substantiated when began with the DL-isomer: \%D in the solution plus precipitate is $49.9 \%$. while it is $44.2 \%$ in the solution alone. and $53 \%$ in the precipitate alone. The heats of reaction for the formation of the different complex isomers did not give definitive results. The $\mathrm{X}$-ray bond lengths and bond angles do not match exactly for the complexes. It is well known that racemization must be enhanced in the presence of transition metal ions such as Co(II) and $\mathrm{Zn}$ (II) ions, but the results indicate retardation of racemization by $\mathrm{Ni}(\mathrm{II})$ ion. In a saturated racemic nixture upon heating for racemization. the formed precipitate is rich of the D-isomer. It should be noted that a careful study of results, however. shows that the deviations were not subjected to an evaluation considering standard errors of the analytical and diffraction methods employed. Extended statistics would be necessary in future to such small deviations to make them meaningful and unequivocal. The data in the tables and elsewhere are generally not assigned error values. Overall, this work rather reminds us of the concept of bond stretch isomerism, introduced by Chatt. ${ }^{2-}$ This was later explained in terms of disorder in the crystal structures. from snall amounts of impurities. Such could not be the case here. and the disorder in the 5-membered rings. noted, night not be consistent with this explaination.

Finally. the homochirality of life material is surely a great mystery to scientists. because enantiomeric couple is considered to have same energy and chemical properties. Inducing chiral

Table 5. Statistically insignificant absorption maxima shifts between $\mathrm{L}$ and $\mathrm{D}$ complexed isomers

\begin{tabular}{cccc}
\hline Peak & D-alanine & L-alanine & $\Delta L-D$ \\
\hline 1 & 366 & 367 & 1 \\
2 & 599 & 602 & 3 \\
\hline
\end{tabular}


preference from achiral environment is certainly a big challenge in current science. Alaninato $\mathrm{Ni}$ (II) diaqua complexes are precipitated in D-form preference under the high temperature and racemization conditions. However. current scientific theory does not support such surprising result. Rather. current science may argue that such results are only due to some errors. In fact. much efforts were made to explain the results. but it was hard to explain what was observed in this work. even though the scientific methods and results seem interesting. solid and valuable to give an impact to the scientific world. However. may be no body can explain the result currently. It should be noted that the experiment was carried out three times. and so it is reproducible. This is important because current science does not expect such a result. even though some scientists are arguing that such result is supported by theories.

\section{Conclusions}

This paper demonestrates results of attempts to influence racemization rates of alanine in supercritical aqueous solution by addition of nickel(II) salts leading finally to surprising and unexpected chiral preference. Concentrations of enantiomers (i.e. the chiral separation) before and after complexation were determined by GC, while UV-Visible spectroscopy was employed for characterizing the complexes. Crystals were investigated by single crystal $\mathrm{X}$-ray diffraction. Enthalpy changes associated with complexation were measured by isothermal calorimetry. There are significant differences between $D$ and $L$ amino acid complexes of $\mathrm{Ni}$ (II) that lead to concentration of the $\mathrm{D}$ isomer in the precipitate and the $\mathrm{L}$ isomer in solution. This result is of importance to the evolution of the stereochemical bias of proteins of biological origin. The results need to be analyzed by a statistical method in near future.

Aknow ledgments. Awni Khatib wishes to thank the CICS and Fulbright (2007-2008) for a sabbatical at the University of California.

\section{References}

1. Smith, G. G.; Khatib, A. A:; Ready, G. S. J. Ant. Chemt. Soc. $1983,105,293$

2. Shinitzky, M: Nudelman, F.; Barda, Y:; Haimovitz, R.; Chen, E.; Deamer, D. W. Origins of Life and Evolution of the Biosphene 2002, 32, 285 .

3. Scolnik, Y: Portnaya, I; Cogan, U.; Tal, S.; Haimovitz, R:; Fridkin, M.; Elitzur, A. C.; Deamer, D. W.; Shinitzky, M. Plys. Chent Chent Phys 2006, 8, 333.

4. Scori, R. I.; Cimpoiasu, V. M.; Popa, R. Astrobiol. $2007,7,733$.

5. Verardo, G. G.; Geatti, P:; Martinuzzi, P; Merli, M:; Toniutti, N. Eur: J. Org. Chem. 2003, 3840.

6. Shinitzky, M; Shvalb, A.; Elitzur, A. C.; Mastai, Y. J. Phys. Chent 2007,111, 1104.

7. Deamer, D. W: Dick, R.; Thiemanu, W.; Shinitzky, M. Chilarity 2007, 19,751.

8. Shinitzky, M; Deamer, D. W. Origins of Life and Evolution of Biosphere 2008, $38,271$.

9. Engel, M. H.; Macko, S. A. Noture 1997, 389, 265.

10. Engel, M. H.: Nagy, B. Kamme 1982, $296,837$.

11. Engel, M. H.: Macko, S. A.: Silfer, J. A. Nanure 1990, 348,47

12. Cronin, J. R.: Pizzarello, S. Science 1997, 275, 951.

13. Weber, A. L. Ongins of Life and Evolution of the Biosphere? 2008, 38, 279.

14. Khatib, A. A.: Engel, M. H. Inorg. Chim fcta 1989, 166, 273.

15. Khatib, A. A., M. S. Thesis, Utah State University, Logan, UT, 1981.

16. Khatib, A. A.: Aqra, F.; Deamer, D. W.: Oliver, A. Research Letrers in horganic Chemistry $2009,1$.

17. Khatib, A. A.; Aqra, F :; Deamer, D. W.; Oliver, A. South Braz. J. Chem. 2008, 16,23.

18. Khatib, A. A.: Aqra, F.; Deamer, D. W.; Oliver, A. J. Argent. Chem. Soc. 2009,97, 1.

19. Teoh, S. G.: Chan, B. T: Fun, H. K.: Kamwaya, M. E. $Z$. Kistallogi: 1987, 181, 199.

20. Tianmin, L.; Cunwang, G.; Yugeng, Z. Cinst. Res. Tech. 1990. $25, \mathrm{~K} 5$.

21. Mostâd, A:; Natarajan, S. Z. Kristallogr. 1987, 178, 263.

22. Chatt, I.: Manoilovic-Muir, L.; Muir, K. W. Chem. Conmm. 1971,655 . 